\title{
Identical amino acid sequence of the aroA(G) gene products of Bacillus subtilis 168 and B. subtilis Marburg strain
}

\author{
Alexander Bolotin, Vladimir Khazak, Nathaly Stoynova, \\ Ksenia Ratmanova, Yurgis Yomantas and Yury Kozlov
}

Author for correspondence: Alexander Bolotin. Tel: +7 09531537 74. Fax: +7 0953150501. e-mail: lab12@vnigen.msk.su

Department of Biotechnology, Research Institute of Genetics and Selection of Industrial Micro-organisms (GNIIgenetika), 1 Dorojhny proezd 1A, 113545

Moscow, Russia

\begin{abstract}
A DNA fragment containing the aroA(G) gene of Bacillus subtilis 168, encoding 3-deoxy-D-arabino-heptulosonate 7-phosphate (DAHP) synthase-chorismate mutase, was cloned and sequenced. The $\mathrm{N}$-terminus of the protein encoded by aroA(G) showed homology with chorismate mutase encoded by aroH of $B$. subtilis and with the chorismate mutase parts of proteins encoded by the pheA and tyrA genes of Escherichia coli. The C-terminus of the aroA(G) product has sequence simililarity with 3-deoxy-D-manno-octulosonate 8-phosphate synthase of $E$. coli. It was shown that the proteins encoded by the aroA(G) gene of B. subtilis 168 and the aroA gene of B. subtilis ATCC 6051 Marburg strain are identical, so the observed differences in DAHP synthase activity from these two strains must result from other changes.
\end{abstract}

Keywords: Bacillus subtilis, aromatic amino acids, chorismate mutase, DAHP synthase
The aromatic amino acids phenylalanine, tyrosine and tryptophan are formed by a sequence of reactions starting with synthesis of 3-deoxy-D-arabino-heptulosonate 7phosphate (DAHP), which occurs by the enzymically catalysed condensation of D-erythrose 4-phosphate and phosphoenolpyruvate (Henner \& Yanofsky, 1993). The synthesis is catalysed by DAHP synthase (EC 4.1.2.15), encoded in Bacillus subtilis by the aro $A$ gene. This gene has been mapped at $265^{\circ}$ on the circular map of the $B$. subtilis chromosome (Anagnostopoulos et al., 1993). DAHP synthase of $B$. subtilis 168 is sensitive to feedback inhibition by chorismate and prephenate and is also responsible for the chorismate mutase activity in this strain (Huang et al., $1974 \mathrm{a}, \mathrm{b})$. The chorismate mutase activity in B.subtilis 168 is assigned to the gene aro $G$ (Hoch \& Nester, 1973). The $\operatorname{aro} A$ and $\operatorname{aro} G$ genes have been placed in one locus on the chromosome map of B. subtilis 168 (Anagnostopoulos $e t$ al., 1993). The enzyme encoded by aro $A(G)$ has been partially purified and data on its amino acid composition obtained (Huang et al., 1974a). It was shown (Llewellyn et al., 1980) that DAHP synthase of B. subtilis 168 was distinct from the monofunctional DAHP synthase of $B$.

Abbreviations: DAHP, 3-deoxy-D-arabino-heptulosonate 7-phosphate. The EMBL accession number for the nucleotide sequence reported in this paper is X65945. subtilis ATCC 6051 Marburg strain. It had an additional chorismate mutase activity, and it was postulated that the bifunctional enzyme arose from the monofunctional enzyme by conversion of an allosteric site for binding prephenate into an active site for chorismate mutase activity (Llewellyn $e t$ al., 1980).

In this paper we present our data on the cloning and structural analysis of the aro $A(G)$ gene of $B$. subtilis 168 and the aro $A$ gene of $B$. subtilis ATCC 6051 Marburg strain.

The aro $A(G)$ gene of $B$. subtilis 168 was cloned on a $2 \mathrm{~kb}$ DNA fragment by partial digestion of the chromosome with Sau3A, using the plasmid pIJ2 (Yomantas et al., $1979)$ as the vector. The selection was carried out by complementation of the aroG932 mutation of an aroG932 leuA8 recE4 strain of B. subtilis (Yu. Yomantas, GNIIgenetika, Moscow). The cloned fragment also complements the aro $A 6$ mutation of $B$. subtilis. The level of DAHP synthase and chorismate mutase activities in crude extracts of a $B$. subtilis strain carrying the aro $A(G)$ fragment on a multicopy plasmid was about 10 times higher than in the recipient $B$. subtilis strain without the plasmid (data not shown).

The nucleotide suquence of the fragment is shown in Fig. 1. It contains two open reading frames, designated orf 324 and orf1102. Both have putative ribosome-binding sites 
gatcaagtcctccagacgaaagaagatgtaaaagacgaactgaaagatgcacaaaaacaggctgagcagttgaatcgctgagcagcoaagraagagcttg 100

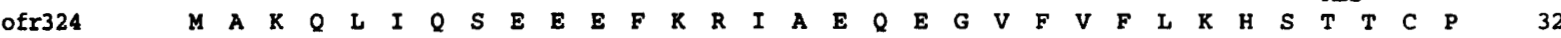
agtcatggctaaacagcttattcaatctgaggaagaatttaaacggattgcagaacaggaaggcgttttcgtcttcttgaaacacagcaccacatgcccg 200

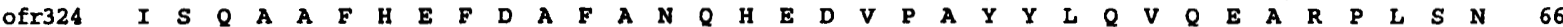
atcagccaggcagcatttcatgagtttgatgcetttgcaaatcagcatgaggatgtgccggcttactatcttcaagtacaagaagcceggccgctgtcca 300

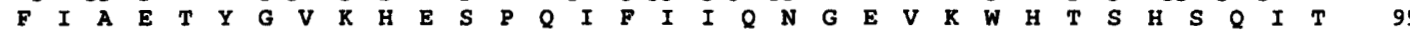
atttcattgcagaaacatatggggtcaagcatgaaagcctcagatattcatcatccagaacggtgaagtgaaatggcatacctcacattcacaaattac 400 E A A $\quad$ I $E$ Q $Q$ H agaagccgctattgagcagcatttgtcatagaaaaaagcgtccagatatcatctggcgcttttttttgtaaaaaacccttgaacattgccctcacatcat 500

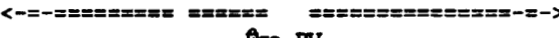

Boo RV

$-35$

gtaggatagcttcatagattactttatcacttaaaagegtttgggtaataaagtagagtttttataaaatttttgtatttcagtaT\%1CCAtttggaat 600 $<=-=-==\mathbf{= = - = - = - = >}$

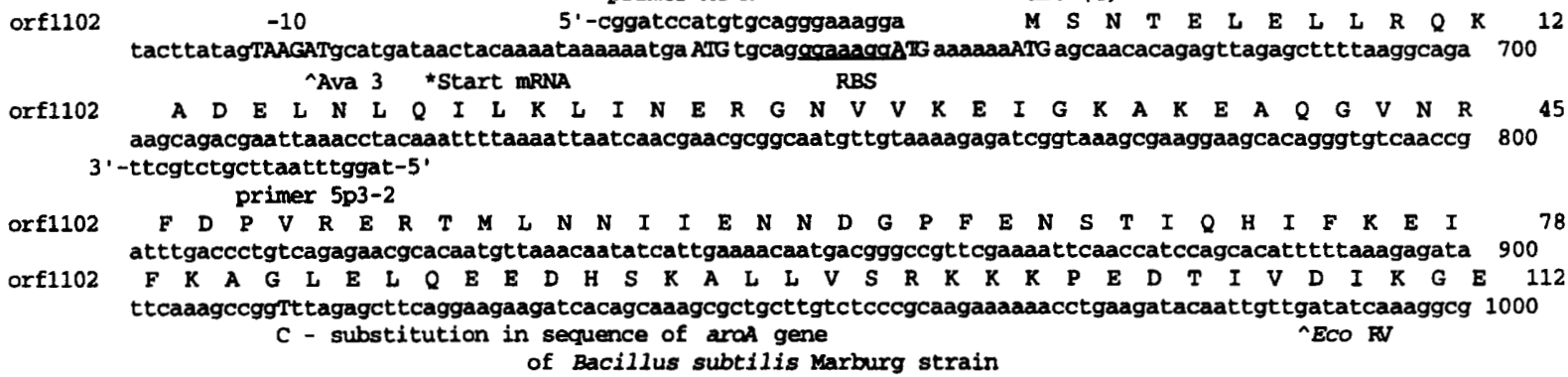

ttataaaaaccaagtatacgttttcatcatctataaaaacgtgtataatttcatgagaagtaattaaatttgatgaataatgaaaaataatgtacactac 1900 tgacttacgcttacaaatcataaacgacataaattcggacattatgacatttctctacataaagtgtttatgctatagataaggataagtgtatccagta 2000 aaaggagtggttttaggatgagcaatattacgatc 2035

Fig. 1. Nucleotide sequence of the cloned DNA fragment. The sites for Avall, EcoRI, EcoRV and Pstl are indicated. The amino acid sequences of the products of orf324 and orf1102 (aroA(G)) are indicated in single-letter code. Paired arrows under the nucleotide sequence denote inverted repeats. The transcriptional start point of aroA(G) mRNA (which was determined in primer extension experiments with oligonucleotide $5 p 3-2$ ) is indicated by an asterisk. The -35 and -10 regions of the promoter and putative ATG start codons of aroA(G) are shown by capital letters. The oligonucleotides AG$\mathrm{N}$ and $\mathrm{AG}-\mathrm{C2}$ were used for synthesis of the PCR product of the aroA gene of $B$. subtilis Marburg strain.

and $\rho$-independent transcription terminator structures. The start point of orf1102 mRNA was determined by primer extension analysis; -35 and -10 regions upstream of it resemble the consensus structure of $\sigma^{A}$ promoters (Fig. 1). The protein encoded by orf1102 has a molecular mass and amino acid composition similar to purified DAHP synthase (Huang et al., 1974a). Disruption of orf1102 by inserting a DNA fragment into the PstI or EcoRI site resulted in the loss of the complementation of the aro $A$ mutation in $B$. subtilis. These data support the identity of orf1102 as the aro $A(G)$ gene.
The amino acid sequence of the $\operatorname{aro} A(G)$ gene product (DAHP synthase-chorismate mutase) was compared with the PIR/NBRF protein data bank. The N-terminal part of the protein (amino acids 1-60) shows homology with the chorismate mutase parts of bifunctional enzymes encoded by the phe $A$ and $\operatorname{tyr} A$ genes of $E$. coli (Hudson \& Davidson, 1984) and monofunctional chorismate mutase encoded by the aroH gene of B. subtilis ATCC 6051 Marburg strain (Gray et al., 1990). Multiple alignments of these proteins are presented in Fig. 2(a). The C-terminal part of the amino acid sequence of DAHP synthase- 
(a)

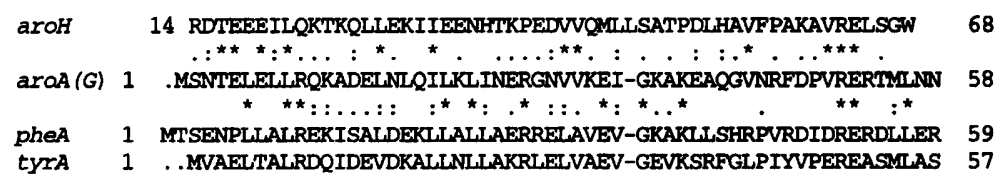

(b)

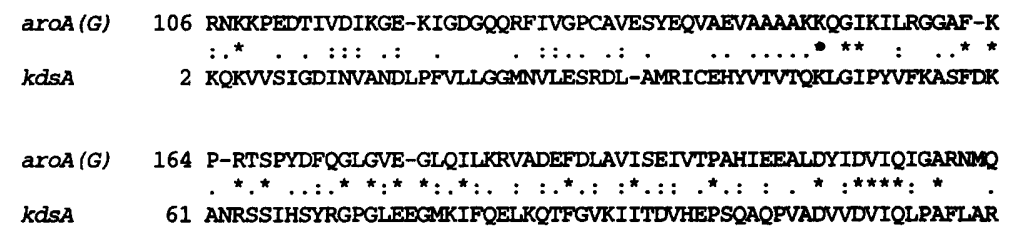

araA (G) 222 NFEILKAAGAVKKPVLLKRGLAATISEFINAAEYIMSQGNDQIILCERGIR-TYETATRN kdSA 121 QTDLVEAMAKTGAVINVKKRPFVSPGQMGNIVDKFKEGGNEKVILCDRGANFGYDNLVVD

arOA (G) 281 TLDISAVPILKQETHILPVFVDVTH-- - - - STGRRDLLLPTAKAALATGADGVMA

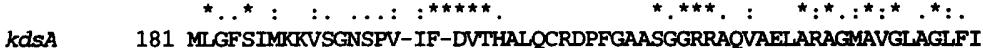

$\operatorname{arOA}(G) \quad 340$ EVHPDPSVALSDSAQQMAIPEFEKWLNELIKPMVKV

$k d S A \quad 239$ EAHPDPEHAKCDGPSALPLAKLEPFLKOMKAIDDL

\begin{abstract}
Fig. 2. Protein sequence alignments of DAHP synthase-chorismate mutase (aroA(G)) encoded by the $\operatorname{aro} A(G)$ gene of $B$. subtilis with: (a) monofuntional chorismate mutase (aroH) encoded by the aroH gene of $B$. subtilis ATCC 6051 Marburg strain (Gray et al., 1990), chorismate mutase-prephenate dehydratase (pheA), chorismate mutaseprephenate dehydrogenase (tyrA) encoded by the phe $A$ and tyrA genes of $E$. coli (Hudson \& Davidson, 1984); and (b) 3-deoxyD-manno-octulosonate 8-phosphate synthase $(k d s A)$ encoded by the $k d s A$ gene of $E$. coli (Woisetschlager \& Hogenauer, 1987). Similarities in amino acid sequences are marked by asterisks (same amino acid), colons (strong similarity) and dots (family similarity). Alignments were done with the DNA-SUN software (A. Mironov, GNIIgenetika).
\end{abstract}

chorismate mutase has no significant similarities to sequences of known E. coli DAHP synthases (Davies \& Davidson, 1982; Schulz et al., 1984; Zurawski et al., 1981), but has some similarity to the amino acid sequence of 3-deoxy-D-manno-octulosonate 8-phosphate synthase of E. coli (EC 4.1.2.16) encoded by the kds $A$ gene (Woisetschlager \& Hogenauer, 1987) (Fig. 2b). This enzyme was responsible for similar aldole condensation of D-arabino 5-phosphate (inside D-erythrose 4-phosphate) and phosphoenolpyruvate (Levin \& Racker, 1959), which resulted in the 3-deoxy-D-manno-octulosonic acid-a sugar which links the hydrophilic polysaccharide chains of the cell wall in Gram-negative bacteria.

The 1116 base PCR product of the aro $A$ gene (amplified with oligonucleotides AG-N and AG-C2: Fig. 1), which encodes monofunctional DAHP synthase in $B$. subtilis Marburg strain, was used for DNA sequencing and was cloned in the BamHI site of plasmid pCB22 (Sorokin \& Khazak, 1990) downstream of expression unit EU19035. The recombinant plasmid complemented aro $A$ and aro $G$ mutations in $B$. subtilis. The complete nucleotide sequence of PCR product was determined; it has only one $\mathrm{T} \rightarrow \mathrm{C}$ substitution at position 912, which does not change the encoded amino acid. Thus the product encoded by the aro $A$ gene of the Marburg strain is the same as that of the $\operatorname{aro} A(G)$ gene of strain 168 . We propose that the different properties described for DAHP synthases isolated from $B$. subtilis 168 and B. subtilis Marburg (Llewellyn et al., 1980; Huang et al., 1974a) could be explained by particularities of the multienzyme complex formed in these two strains and mimic effects of high active chorismate mutase encoded by aroH in the Marburg strain.

\section{REFERENCES}

Anagnostopoulos, C., Piggot, P. J. \& Hoch, J. A. (1993). The genetic map of Bacillus subtilis. In Bacillus subtilis and Other Grampositive Bacteria, pp. 425-461. Edited by A. L. Sonenshein, J. A. Hoch \& R. Losick. Washington, DC: American Society for Microbiology.

Davies, W. D. \& Davidson, B. E. (1982). The nucleotide sequence of aroG, the gene for 3-deoxy-D-arabinoheptulosonate 3-phosphate synthase(phe) in Escherichia coli K-12. Nucleic Acids Res 10, 4045-4058.

Gray, J. V., Golinelli-Pimpaneau, B. \& Knowles, J. R. (1990). Monofunctional chorismate synthase from Bacillus subtilis: purification of the protein, molecular cloning of the gene, and overexpression of the gene product in Escherichia coli. Biochemistry 29, 376-383.

Henner, D. \& Yanofsky, C. (1993). Biosynthesis of aromatic amino acids. In Bacillus subtilis and Other Gram-positive Bacteria, pp. 269-280. Edited by A. L. Sonenshein, J. A. Hoch \& R. Losick. Washington, DC: American Society for Microbiology.

Hoch, J. A. \& Nester, E. W. (1973). Gene-enzyme relationships of aromatic acid biosynthesis in Bacillus subtilis. J Bacteriol 116, 59-66.

Huang, L., Nakatsukasa, W. \& Nester E. W. (1974a). Regulation of aromatic amino acid biosynthesis in Bacillus subtilis 168 : purification, characterization and subunit structure of the bifunctional enzyme 3deoxy D-arabinoheptulosonate 7-phosphate synthetase-chorismate mutase. J Biol Chem 249, 4467-4472.

Huang, L., Montoya, A. \& Nester, E. W. (1974b). Characterization of the functional activities of the subunits of 3-deoxy D-arabinoheptulosonate 7-phosphate synthetase-chorismate mutase from Bacillus subtilis 168. J Biol Chem 249, 4473-4479.

Hudson, G. S. \& Davidson, B. E. (1984). Nucleotide sequence and transcription of the phenylalanine and tyrosine operons of Eschericbia coli K-12. J Mol Biol 180, 1023-1051. 
Levin, D. H. \& Racker, E. (1959). Condensation of D-arabinose 5phosphate and phosphorylenol pyruvate by 2-keto-3-deoxy-8phosphooctonic acid synthetase. J Biol Chem 234, 2532-2539.

Llewellyn, D., Daday, A. \& Smith G. (1980). Evidence for artificially evolved bifunctional 3-deoxy D-arabinoheptulosonate 7-phosphate synthase-chorismate mutase in Bacillus subtilis. J Biol Chem 255, 2077-2084.

Schulz, J., Hermodson, M. A., Garner, C. C. \& Herrman, K. M. (1984). The nucleotide sequence of the aroF gene of Escherichia coli and the amino acid sequence of the encoded protein, the tyrosine sensitive 3-deoxy-D-arabinoheptulosonate 3-phosphate synthase. $J$ Biol Chem 259, 9655-9661.

Sorokin, A. V. \& Khazak, V. E. (1990). Expression unit in region of replication initiation in plasmid pSM19035 of streptococci. Molecularnaya Biologia (in Russian) 24, 993-1000.
Woisetschlager, M. \& Hogenauer, G. (1987). The $k d s A$ gene coding for 3-deoxy-D-manno-octulosonic acid 8-phosphate synthetase is part of an operon in Escherichia coli. Mol \& Gen Genet 207, 369-375.

Yomantas, Yu. V., Rabinovitch, P. M., Bandrin, S. V., Demjanova, N. G., Kozlov, Yu. I. \& Stepanov, A. I. (1979). Bireplicon vectors for bacterial system: Escherichia coli-Bacillus subtilis. Dokl Akad Nauk SSSR 225, 993-996.

Zurawski, G., Gunsalus, R. P., Brown, K. D. \& Yanofsky, C. (1981). Structure and regulation of aroH, the structural gene for tryptophane repressible 3-deoxy-D-arabinoheptulosonate 3-phosphate synthetase of Escherichia coli. J Mol Biol 145, 47-73.

Received 3 April 1995; accepted 11 May 1995. 